\title{
Equation of State, Spectra and Composition of Hot and Dense Infinite Hadronic Matter in a Microscopic Transport Model*
}

\author{
M. Belkacem ${ }^{1 \dagger}$, M. Brandstetter ${ }^{1}$, S. A. Bass ${ }^{2 \ddagger}$, M. Bleicher ${ }^{1 \S}$, L. Bravina $^{1 \dagger}$, \\ M. I. Gorenstein ${ }^{3}$, J. Konopka ${ }^{1}$, L. Neise ${ }^{1}$, C. Spieles ${ }^{1}$, S. Soff ${ }^{1}$, H. Weber ${ }^{1}$, \\ H. Stöcker ${ }^{1}$ and W. Greiner ${ }^{1}$ \\ ${ }^{1}$ Institut für Theoretische Physik, J. W. Goethe-Universität, \\ D-60054 Frankfurt am Main, Germany \\ 2 Dept. of Physics, Duke University, Durham 27708-0305, USA \\ 3 Bogolyubov Institute for Theoretical Physics, Kiev, Ukraine
}

(October 24, 2018)

\begin{abstract}
Equilibrium properties of infinite relativistic hadron matter are investigated using the Ultrarelativistic Quantum Molecular Dynamics (UrQMD) model. The simulations are performed in a box with periodic boundary conditions. Equilibration times depend critically on energy and baryon densities. Energy spectra of various hadronic species are shown to be isotropic and consistent with a single temperature in equilibrium. The variation of energy density versus temperature shows a Hagedorn-like behavior with a limiting temperature of $130 \pm 10 \mathrm{MeV}$. Comparison of abundances of different particle species to ideal hadron gas model predictions show good agreement only if detailed balance is implemented for all channels. At low energy densities, high mass resonances are not relevant; however, their importance raises with increasing energy density. The relevance of these different conceptual frameworks for any interpretation of experimental data is questioned.
\end{abstract}

\footnotetext{
*supported by BMBF, GSI, DFG and Graduiertenkolleg 'Schwerionenphysik'

$\dagger$ Alexander von Humboldt Fellow

${ }^{\ddagger}$ Feodor Lynen Fellow of the Alexander von Humboldt Foundation

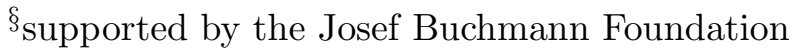


The intriguing possibilities of phase transitions at various orders in the nuclear equation of state (EoS) have long played a central role in heavy-ion physics from intermediate to very high energies [1] 3]. Indications about the nuclear liquid-gas phase transition at moderate temperatures, $T<10 \mathrm{MeV}$, and densities, $\rho<\rho_{0}$, 回 8 , and about the transition to resonance matter at higher densities and/or temperatures $\left(\rho \approx 3-5 \rho_{0}, T \approx 100-150 \mathrm{MeV}\right)$ [9 11] have been reported. Deconfinement of hadronic matter into quark-gluon plasma and strange matter may occur at even higher energy densities. Deconfinement temperatures of about $T_{c} \approx 150 \pm 10 \mathrm{MeV}$ have been predicted by lattice QCD simulations for zero net baryon - and strangeness density [12]. However, the statistical concept of phase transitions is developed for stationary states close to equilibrium [13 [16]. Therefore, the question immediately arises: can thermal and chemical equilibrium concepts actually be used and equilibrium be reached in central heavy-ion collisions?

At bombarding energies $E_{\mathrm{Lab}} \leq 1 \mathrm{GeV} /$ nucleon, experimental data on multifragmentation [17] have been described e.g. by statistical models (Quantum Statistical Model (QSM) [18 20] or Statistical Multifragmentation Models (SMM) [21]). Also measured particle abundances and energy spectra from central heavy-ion reactions at both AGS (14.6 GeV/nucleon) and SPS (200 GeV/nucleon) energies [22] have been described in statistical models assuming global thermal and hadrochemical equilibrium (with overall moderate longitudinal and transverse expansions) [23 26]. These models assume an instantaneous global freeze-out for all particles.

However, this hypothesis is - on first sight - not consistent with the results of microscopic models. They predict different freeze-out times and radii for the different particle species [27 31]. An other related question is, whether the short time scales $\left(10^{-23} \mathrm{~s}\right)$ are long enough for the system to move through consecutive equilibrium states before freeze-out.

In this paper, we study equilibrium properties of infinite relativistic hadronic matter within the framework of the Ultrarelativistic Quantum Molecular Dynamics (UrQMD) model [29]. However, in contrast to the usual simulations of heavy-ion collisions from the two colliding nuclei to the complex final state, here a closed microcanonical system is constructed at a fixed net baryon density and net strangeness density, confined to a cubic box with periodic boundary conditions. The yield of the various particle species can change due to inelastic collisions and decays of hadron resonances and strings.

UrQMD, as a dynamical microscopic model, follows the time evolution of a nonequilibrium A-particle system, e.g. a heavy-ion reaction, in the entire many-body phase space. It has been applied in the energy range from $100 \mathrm{MeV} /$ nucleon up to several hundreds of $\mathrm{GeV} /$ nucleon for heavy ion collisions using the same basic concepts and physics inputs at all energies. The model includes explicitly 55 different baryon species (nucleons, deltas, hyperons, and their known resonances [32] up to masses of $2.25 \mathrm{GeV}$ ) and 32 different meson species (including the known meson resonances [32] up to masses of $2 \mathrm{GeV}$ ), as well as their respective anti-particles and all isospin-projected states [29]. For higher mass excitations, a string mechanism is invoked. Hadrons produced through string decays have non-zero formation time $\tau_{f}$, which depends on energy-momentum of the particle. Newly formed particles cannot interact during their formation time. The leading hadrons with constituent quarks interact within their formation time with a reduced cross section, which is taken to be proportional to the number of their original constituent quarks. All hadrons are propagated (in a relativistic cascade sense) according to Hamilton's equations of mo- 
tion, supplemented by a relativistic Boltzmann-Uehling-Uhlenbeck collision term involving all hadron states. The collision term is based on tabulated or parameterized experimental cross section (when available). Resonance absorption and scattering is handled via the principle of detailed balance. If no experimental information is available, the cross section is either calculated via an OBE model or via a modified additive quark model. The baryonantibaryon annihilation cross section is parameterized as the proton-antiproton annihilation cross section at the same center of mass energy. For a detailed description of the UrQMD model, the reader is referred to the original publication of the model presentation [29].

For the present study, additional features have been added to the model, which allow for the calculation of infinite hadronic matter properties in equilibrium. This is done by confining the different particles in a cubic box with cyclic boundary conditions at a fixed net baryon density $\rho_{B}$ and fixed net strangeness $\rho_{S}$. In this paper, we restrict our study to a net baryon density $\rho_{B}=\rho_{0}=0.16 \mathrm{fm}^{-3}$ and $\rho_{S}=0 \mathrm{fm}^{-3}$, i.e. net strangeness zero. Although in heavy-ion collisions, $\rho_{S} \neq 0$ and $\rho_{B} \neq \rho_{0}$ are expected, we shift the detailed study of different net baryon densities and/or non zero net strangeness to a forthcoming paper [33].

The initial system considered here consists of 80 protons +80 neutrons, which are uniformly distributed in configuration space in a cubic box of $10 \times 10 \times 10 \mathrm{fm}^{3}$. The momenta are uniformly distributed in a sphere with random radius and then rescaled to the desired energy density.

Figure 1 displays the time evolution of the multiplicities (averaged over 50 events) of nucleons, pions and kaons at two different energy densities, $\epsilon=200 \mathrm{MeV} / \mathrm{fm}^{3}$ (upper panel) and $\epsilon=700 \mathrm{MeV} / \mathrm{fm}^{3}$ (lower panel). Particle multiplicities saturate after some time. This equilibration time depends strongly on energy density $\epsilon$. The different hadrons continue to interact strongly, but their average absolute numbers remain nearly constant. Note that the equilibration time of kaons is much longer at the energy density $\epsilon=0.2 \mathrm{GeV} / \mathrm{fm}^{3}$ than those for nucleons and pions, which are rather similar. In fact, this is the case for all strange hadrons (both mesons and baryons). This difference between the strange and non strange hadrons decreases with growing energy density. Equilibration times of all hadrons are quite similar for energy densities above $0.7 \mathrm{GeV} / \mathrm{fm}^{3}$.

We have checked whether our system is ergodic in the equilibrium phase: Both ensemble averages (i.e. when the averaging is done over a large set of parallel events) and time averages (when the averaging is done over time evolution of a single event, but in both cases after equilibrium is established) coincide within $1 \%$. The following results are obtained by time averaging (if not stated otherwise). All quantities presented below have been extracted from the equilibrium phase, i.e. after particle multiplicities have achieved their saturation valuest Figure 2 shows the energy spectra for four particle species; nucleons, pions, lambdas and kaons (top panels) for two different energy densities $\epsilon=250 \mathrm{MeV} / \mathrm{fm}^{3}$ (left) and $\epsilon=800$

\footnotetext{
${ }^{1}$ In practice, we start the time averaging much after particle multiplicities of the most relevant species $(\mathrm{N}, \pi, \Delta, \mathrm{K}, \Lambda)$ have achieved their saturation values, to allow for the equilibration of the higher resonances. E.g., at $\epsilon=0.2 \mathrm{GeV} / \mathrm{fm}^{3}$, the averaging starts after a time evolution of 500 $\mathrm{fm} / \mathrm{c}$, much after the kaons have equilibrated $(\sim 250 \mathrm{fm} / \mathrm{c}$, see Fig. 1). For energy densities larger than $0.5 \mathrm{GeV} / \mathrm{fm}^{3}$, we average after an initial evolution of $150 \mathrm{fm} / \mathrm{c}$. Moreover, in most cases, the time averaging is done for a time evolution from 500 to $1000 \mathrm{fm} / \mathrm{c}$.
} 
$\mathrm{MeV} / \mathrm{fm}^{3}$ (right). The distributions of the three momentum components $p_{x}, p_{y}$ and $p_{z}$ are given for nucleons in the bottom panels. The energy spectra (top panels) are reproduced by Boltzmann distributions, $\exp (-E / T)$, with nearly the same temperature for all 4 species $\left(T=125 \pm 10 \mathrm{MeV}\right.$ for $\epsilon=250 \mathrm{MeV} / \mathrm{fm}^{3}$, and $T=130 \pm 10 \mathrm{MeV}$ for $\left.\epsilon=800 \mathrm{MeV} / \mathrm{fm}^{3}\right)$.

The momentum components distributions (lower part of the figure) are reproduced by Gaussian distributions, $\exp \left(-p^{2} / 2 m T\right)$, with the same temperature for the three space directions (complete isotropy of the momentum distributions). This holds for all other hadron species as well, if analogous procedures are applied. At this point, it should be noted that the temperature of mesons is always smaller (by 5 to $10 \%$ ) than that of the baryons. This is mainly due to decay kinematics of resonances [34]. The temperature used in Fig. 2 is the average value between the two. Apart from this small discrepancy, all particle have the same temperature $T$ and are globally equilibrated.

Figure 3 shows the energy density versus the temperature as resulting from the box calculations using the UrQMD model (open diamonds). It shows a Hagedorn-like equation of state [35]: a rapid rise of the temperature at low energy densities is followed by a saturation at a temperature around $130 \pm 10 \mathrm{MeV}$. The calculations have been done up to energy densities of $5 \mathrm{GeV} / \mathrm{fm}^{3}$, where still the same limiting temperature is observed. In the same figure, solid circles show the EoS of the UrQMD model without strings and many-body decays. In this case, the temperature shows a continuous rise with energy density and no limiting temperature is observed. The inclusion of strings in the UrQMD model changes then the equation of state from a continuously increasing temperature with energy density (when strings are not taken into account) to a Hagedorn-like EoS with a limiting temperature, $T_{H}=130 \pm 10 \mathrm{MeV}$.

Ideal hadron gas models have been used to describe particle abundances and energy spectra from central heavy-ion reactions at both AGS and SPS energies [23] 26]. In these models, the system is described by a grand canonical ensemble of non-interacting fermions and bosons in equilibrium at temperature $T$. Particle multiplicities are given by

$$
N_{i}=\frac{g_{i} V}{(2 \pi \hbar)^{3}} \int_{0}^{\infty} \frac{4 \pi p^{2} d p}{\exp \left[\left(E_{i}-B_{i} \mu_{B}-S_{i} \mu_{S}\right) / T\right] \pm 1} .
$$

Here $g_{i}$ is the spin-isospin degeneracy factor of particle $i, E_{i}, B_{i}$ and $S_{i}$ are the single particle energy, baryon number and strangeness, and $\mu_{B}$ and $\mu_{S}$ are the baryon and strangeness chemical potentials (the electric chemical potential has been neglected). $V$ is the volume of the box. For resonances with a finite width, Eq.(11) is folded by an integration over the mass distribution $\rho_{i}(m)$ :

$$
N_{i}=\frac{g_{i} V}{(2 \pi \hbar)^{3}} \int \rho_{i}(m) d m \int_{0}^{\infty} \frac{4 \pi p^{2} d p}{\exp \left[\left(E_{i}(m)-B_{i} \mu_{B}-S_{i} \mu_{S}\right) / T\right] \pm 1},
$$

For $\rho_{i}(m)$, the Breit-Wigner mass distribution of resonance $i$ is used. All hadron species used in the UrQMD model [29] have been included in the present statistical model. The UrQMD model uses a stochastic collision term, and soft or hard core effects are neglected for all different particles (cascade calculations). Hence, excluded volume (Van der Waals) correction [26] has not been included into Eqs.([1.22). The excluded volume decreases the energy density and changes the EoS as well as transport coefficients considerably in the high density limit. The inclusion into a relativistic transport theory in nontrivial, the non-relativistic 
Chapmann-Enskog expansion cannot simply be extended due to causality problems arising in the relativistic case.

The strategy adopted to determine the three parameters of the statistical model ( $T$, $\mu_{B}$, and $\left.\mu_{S}\right)$ is the following: Instead of fitting these parameters to some particular particle multiplicities and energy spectra [23,25,26], the three parameters of the statistical model are defined by the input used for the corresponding UrQMD box calculations. For fixed energy density, baryon density and strangeness density, the parameters are defined such that:

$$
\begin{aligned}
\epsilon & =\sum_{i} \frac{g_{i}}{(2 \pi \hbar)^{3}} \int_{0}^{\infty} \frac{E_{i}(m) 4 \pi p^{2} d p}{\exp \left[\left(E_{i}(m)-B_{i} \mu_{B}-S_{i} \mu_{S}\right) / T\right] \pm 1} ; \\
\rho_{B} & =\sum_{i} B_{i} \frac{N_{i}}{V} ; \\
\rho_{S} & =\sum_{i} S_{i} \frac{N_{i}}{V},
\end{aligned}
$$

Here $i$ runs over all hadron species (and their anti-particles). The temperature varies with the energy density obtained in this model (at $\rho_{B}=0.16 \mathrm{fm}^{-3}$ and $\rho_{S}=0 \mathrm{fm}^{-3}$ ) as shown by the solid curve in Fig. 3. The curve exhibits a continuous rise of the temperature with the energy density, in good agreement with UrQMD box calculations without strings and many-body decays (solid circles).

However, in the above sums, string degrees of freedom (which can be considered as heavy mass resonances with small life times) are not taken into account. Therefore, a direct comparison with the UrQMD model (which includes these degrees of freedom) shall yield different results at high energy densities. The same results from the UrQMD box model and the corresponding statistical model can only be expected if strings (or higher mass resonances) are included in the statistical model. For this, the Hagedorn mass spectrum [35] for the strings given by:

$$
\rho^{(s)}(m)=\rho_{0}^{(s)} m^{a_{H}} \exp \left(m / T_{H}\right)
$$

must be included. We fix the Hagedorn temperature $T_{H}$ to the limiting temperature obtained in standard UrQMD box calculations, $T_{H}=130 \mathrm{MeV}$. Moreover, the constants $\rho_{0}^{(s)}$ and $a_{H}$ have been fixed such that to obtain the same equation of state $\epsilon(T)$ as in the UrQMD box model (open diamonds in Fig. 3). Their values are: $\rho_{0}^{(s)}=500 \mathrm{MeV}^{2}$ and $a_{H}=-3$.

Six different kinds of strings are excited within the UrQMD model, namely baryon strings with different strangeness content $(B=1$ and $S=0,-1,-2,-3)$ and meson strings with strangeness 0 or $1(B=0$ and $S=0,1)$ and their respective anti-strings (anti-baryon strings, $B=-1$ and $S=0,1,2,3$ and anti-meson string, $B=0$ and $S=-1$ ). String multiplicities are then given by:

$$
N_{j}^{(s)}=\frac{V}{(2 \pi \hbar)^{3}} \int_{m_{\text {min }}}^{\infty} \rho^{(s)}(m) d m \int_{0}^{\infty} \frac{4 \pi p^{2} d p}{\exp \left[\left(E_{j}(m)-B_{j} \mu_{B}-S_{j} \mu_{S}\right) / T\right] \pm 1},
$$

where the index $j, j=1 \ldots 6$, distinguishes the six kinds of string defined above (plus their respective anti-strings). The lowest mass limit in the mass integration is fixed to $m_{\min }=1.7$ $\mathrm{GeV}$, the lowest mass of the strings excited in UrQMD calculations. The upper limit is 
fixed to $m_{\max }=1000 \mathrm{GeV}$. The variation of the temperature with the energy density in the statistical model of hadrons and strings (discrete and continuous mass spectrum, respectively) is shown in Fig. 3 by the dashed line.

Figure 4 shows particle multiplicities of all baryon (top panel) and meson (lower panel) species as obtained in UrQMD box calculation at $\epsilon=1 \mathrm{GeV} / \mathrm{fm}^{3}, \rho_{B}=0.16 \mathrm{fm}^{-3}$ and $\rho_{S}=0$ $\mathrm{fm}^{-3}$ (club symbols). Also shown are hadron multiplicities resulting from the statistical model where the continuum mass spectrum has been taken into account according to Eq. (6) (open circles).

From Eqs. (3-5), with the sums extended to include string degrees of freedom, we get from the statistical model the following values for the intensive thermodynamical variables: $T=129.98 \mathrm{MeV}, \mu_{B}=505 \mathrm{MeV}$ and $\mu_{S}=130 \mathrm{MeV}$. Large discrepancies are observable between the UrQMD box model and the statistical model for almost all hadron species. The statistical model overestimates almost all baryon multiplicities (e.g. by a factor 5 for nucleons), whereas it underestimates drastically meson multiplicities (almost by an order of magnitude for pions).

It appears that the difference between the two models is due to the principle of detailed balance, which is violated when strings and many-body decays of resonances (more than two outgoing particles) are included into the UrQMD box calculations. It is obvious that detailed balance (which is assumed in the statistical model) must be violated in many-body decays, if for the back reaction only binary collisions are considered, as in the microscopic UrQMD simulation, which employs a Boltzmann limit collision kernel (with molecular chaos). Chapmann-Enskog type extensions would exaggerate this effect even more. String and many-body decays lead to an enhancement of light mesons, mostly pions, resulting in non-zero effective chemical potentials.

Figure 5 shows hadron abundances as obtained in UrQMD box simulations (club symbols) if string degrees of freedom are excluded. At the same time, also all other many-body $(n>2)$ decays are suppressed. The results of the corresponding statistical model are shown in the same figure by open circles for $T=184 \mathrm{MeV}, \mu_{B}=67 \mathrm{MeV}$ and $\mu_{S}=48 \mathrm{MeV}$ from Eqs. (3-5). The sums in the equations run only over hadron degrees of freedom without the continuous mass spectrum. Good agreement between the two models is observed, although there are still some differences for the heaviest resonances. The particles with the highest production probabilities (nucleons, deltas, pions, kaons, etc...) however, agree much better than in Fig. 4. Note that $\eta^{\prime}-$ and $\mathrm{f}_{1}$-mesons are missing in Fig. 5. This is because these particles can be produced in UrQMD simulations only via string decays, which have now been explicitly canceled.

Energy spectra of the particles shown in Fig. 6 are also nicely reproduced a temperature of $180 \mathrm{MeV}$. This $T$-value is in agreement with the temperature obtained in the statistical model for the same input $\epsilon, \rho_{B}$ and $\rho_{S}$ (see above). The dependence of the temperature on the energy density as obtained in the UrQMD simulations, but without strings and manybody decays, is shown in Fig. 3 by the solid circles. The UrQMD model and the statistical model of ideal hadrons seem to agree when strings and many-body decays are excluded in the UrQMD model. The inclusion of strings and many-body decays of resonances results in a Hagedorn-like equation of state.

At this point, a comment is due. It is evident for both models (UrQMD and the statistical model) that string degrees of freedom (a continuously increasing mass spectrum in the 
statistical model) become increasingly relevant with increasing energy density. As an example, at $\epsilon=1 \mathrm{GeV} / \mathrm{fm}^{3}$ and $\rho=0.16 \mathrm{fm}^{-3}$, both models show that an important fraction of the energy density and of the net baryon density is stored in string degrees of freedom. These string degrees of freedom must therefore be taken into account for interpreting heavy-ion experimental data. To date, this has been totally ignored.

In conclusion, we have studied equilibrium properties of relativistic hadronic matter in the framework of the UrQMD model. The system is confined to a box with periodic boundary conditions at fixed baryon density and zero net strangeness. Starting from random initial conditions, particle multiplicities saturate after some time, indicating chemical equilibration. Strange hadrons show much longer equilibration times than non-strange hadrons at low energy densities. This difference, however, disappears at energy densities $\epsilon>500$ $\mathrm{MeV} / \mathrm{fm}^{3}$. The slopes of all hadron spectra can be reproduced by Boltzmann fits with two temperatures $T_{B}$ and $T_{M}$, indicating a complete thermalization of the system with two-body decay contributions. These equilibrium properties allow us to study local thermodynamical equilibrium in realistic heavy-ion collisions 36.

The equation of state of the hot, dense hadron medium (energy density versus temperature) is extracted from the UrQMD model. The EoS appears to be Hagedorn-like, with a limiting temperature of about $T_{H}=130 \pm 10 \mathrm{MeV}$. When comparing the UrQMD results with those resulting from a statistical model for ideal hadrons based on the grand canonical ensemble, the two models agree much better (for hadron multiplicities and energy spectra) when strings and other many-body decays are suppressed in the UrQMD model. The differences when strings and many-body decays are included, are quite pronounced $(>50 \%)$ at energy densities $\epsilon>500 \mathrm{MeV} / \mathrm{fm}^{3}$. This is due to the Boltzmann collision kernel with its restriction to binary collisions in the UrQMD model. It results in a violation of the principle of detailed balance, which is the basis of the statistical model when strings and many-body decays are taken into account. On the other hand, strings are needed for a description of relativistic heavy-ion data in terms of subsequent hadron-hadron collisions (and even for simple first collision models) 29]. The strings carry a substantial fraction of the energy density and the baryon density. Therefore, they should be taken into account in thermal models for comparisons to experimental data in relativistic heavy-ion collisions.

[1] H. Stöcker and W. Greiner, Phys. Rep. 137, 277 (1986).

[2] H. R. Jaqaman, A. Z. Mekjian and L. Zamick, Phys. Rev. C 27, 2782 (1983); Phys. Rev. C 29, 2067 (1984).

[3] A. L. Goodman, J. I. Kapusta and A. Z. Mekjian, Phys. Rev. C 30, 851 (1984).

[4] J. B. Elliott, M. L. Gilkes, J. A. Hauger, A. S. Hirsch, E. Hjort, N. T. Porile, R. P. Scharenberg, B. K. Srivastava, M. L. Tincknell and P. G. Warren, Phys. Rev. C 49, 3185 (1994); M. L. Gilkes et al., Phys. Rev. Lett. 73, 1590 (1994).

[5] J. Pochodzalla et al., Phys. Rev. Lett. 75, 1040 (1995).

[6] P. F. Mastinu et al., Phys. Rev. Lett. 76, 2646 (1996).

[7] M. Belkacem, V. Latora and A. Bonasera, Phys. Rev. C 52, 271 (1995); P. Finocchiaro, M. Belkacem, T. Kubo, V. Latora and A. Bonasera, Nucl. Phys. A600, 236 (1996). 
[8] G. Peilert, J. Randrup, H. Stöcker and W. Greiner, Phys. Lett. B260, 271 (1991).

[9] G. F. Chaplin, M. H. Johnson, E. Teller and M. S. Weiss, Phys. Rev. D 8, 4302 (1973).

[10] H. Stöcker, W. Greiner and W. Scheid, Z. Phys. A286, 121 (1978).

[11] J. Boguta, Phys. Lett. B109, 251 (1981).

[12] C. DeTar, Nucl. Phys. B42, 73 (1995).

[13] L. D. Landau and E. M. Lifshits, Statistical Physics (Pergamon, London, 1958).

[14] R. Balescu, Equilibrium and Nonequilibrium Statistical Mechanics (Krieger Publishing Company, Malabar, Florida, 1991).

[15] K. Huang, Statistical Mechanics (John Wiley \& Sons, New York, 1987).

[16] W. Greiner, L. Neise and H. Stöcker, Thermodynamics and Statistical Mechanics (SpringerVerlag, New York, 1997).

[17] M. D'Agostino et al., Phys. Rev. Lett. 75, 4373 (1995); Phys. Lett. B368, 259 (1995).

[18] D. Hahn and H. Stöcker, Nucl. Phys. A476, 718 (1988).

[19] J. Konopka, H. Graf, H. Stöcker and W. Greiner, Phys. Rev. C 50, 2085 (1994).

[20] F. Gulminelli and D. Durand, Nucl.Phys. A615, 117 (1997).

[21] D. H. E. Gross, Prog. Nucl. Phys. 30, 155 (1993); J. Bondorf et al, Phys. Rep. 257, 133 (1995), and references therein.

[22] Proceedings of Quark Matter 96, Nucl. Phys. A610 (1996).

[23] J. Cleymans and H. Satz, Z. Phys. C 57, 135 (1993); J. Cleymans, K. Redlich, H Satz and E. Suhonen, Z. Phys. C 58, 347 (1993); Nucl. Phys. A566, 391c (1994).

[24] J. Letessier, J. Rafelski and A. Tounsi, Phys. Lett. B321, 394 (1994).

[25] P. Braun-Munzinger et al., Phys. Lett. B344, 43 (1995); Phys. Lett. B365, 1 (1996).

[26] G. D. Yen, M. I. Gorenstein, W. Greiner and S. N. Yang, Phys. Rev C 56, 2210 (1997).

[27] L. V. Bravina et al., Phys. Lett. B354, 196 (1995); Heavy Ion Phys. 5, 455 (1997).

[28] S. A. Bass et al., nucl-th/9711032, submitted to Phys. Rev. Lett. (1998).

[29] S. A. Bass, M. Belkacem, M. Bleicher, M. Brandstetter, L. Bravina, C. Ernst, L. Gerland, M. Hofmann, S. Hofmann, J. Konopka, G. Mao, L. Neise, S. Soff, C. Spieles, H. Weber, L. A. Winckelmann, H. Stöcker, W. Greiner, Ch. Hartnack, J. Aichelin and N. Amelin, nuclth/9803035, Progr. Part. Nucl. Phys. 41 (1998), in press.

[30] H. Sorge, Phys. Lett. B373, 16 (1996).

[31] M. Bleicher et al., Proceedings of the Winter Meeting in Nuclear Physics (1997), Bormio (Italy), nucl-th/9704065; S. Soff et al., to be submitted for publication.

[32] Particle-Data-Group, Phys. Rev. D 54 (1996).

[33] M. Belkacem et al., in preparation.

[34] M. Brandstetter et al., to be submitted for publication.

[35] R. Hagedorn, Suppl. Nuovo Cimento 3, 147 (1965); Suppl. Nuovo Cimento 6, 311 (1965); R. Hagedorn and J. Ranft, Suppl. Nuovo Cimento 6, 169 (1968).

[36] L. V. Bravina et al., nucl-th/9804008, submitted for publication to Phys. Lett. B (1998). 

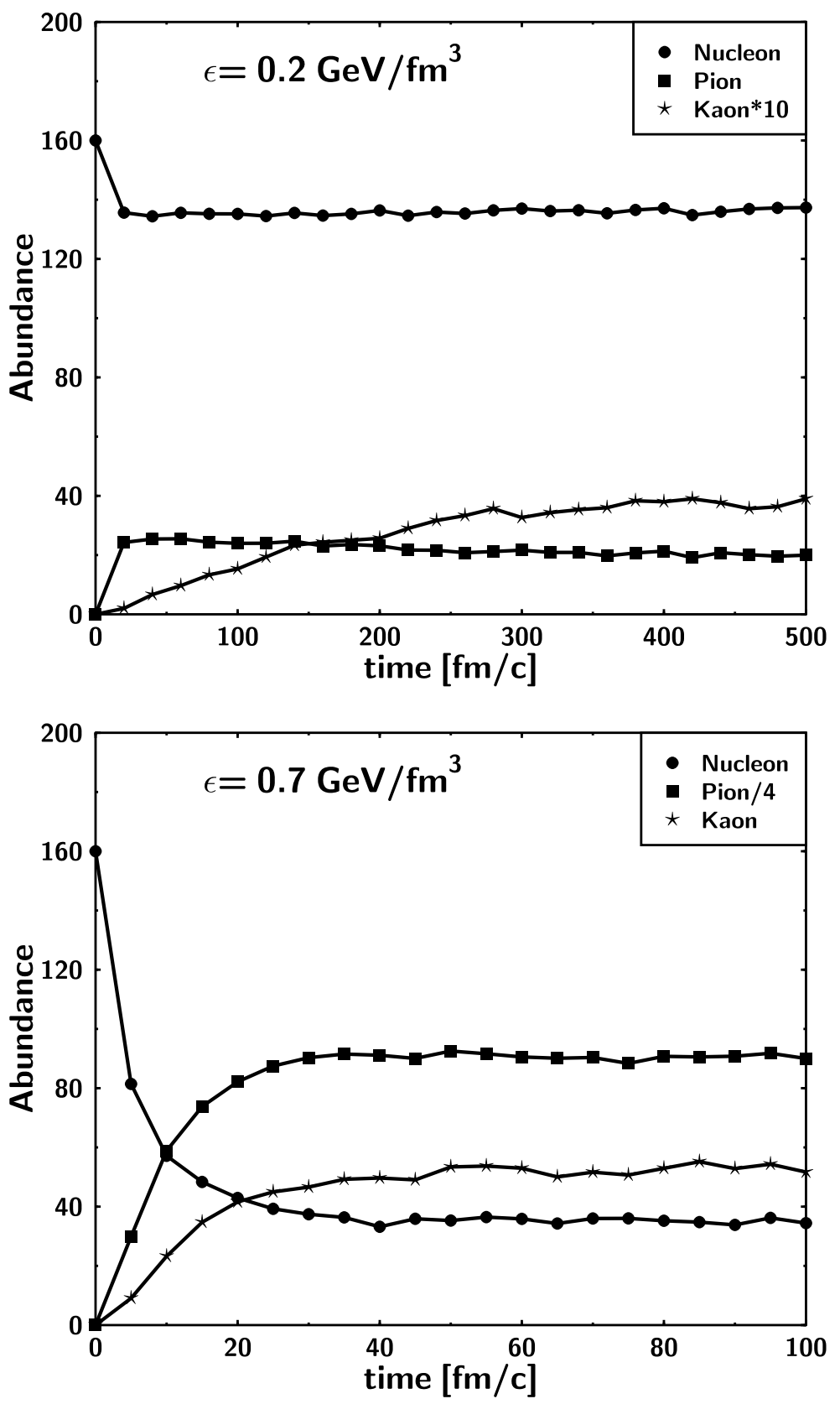

FIG. 1. Time evolution of particle multiplicities for two different energy densities. The calculations are done at $\rho_{B}=0.16 \mathrm{fm}^{-3}$ and $\rho_{S}=0 \mathrm{fm}^{-3}$. 

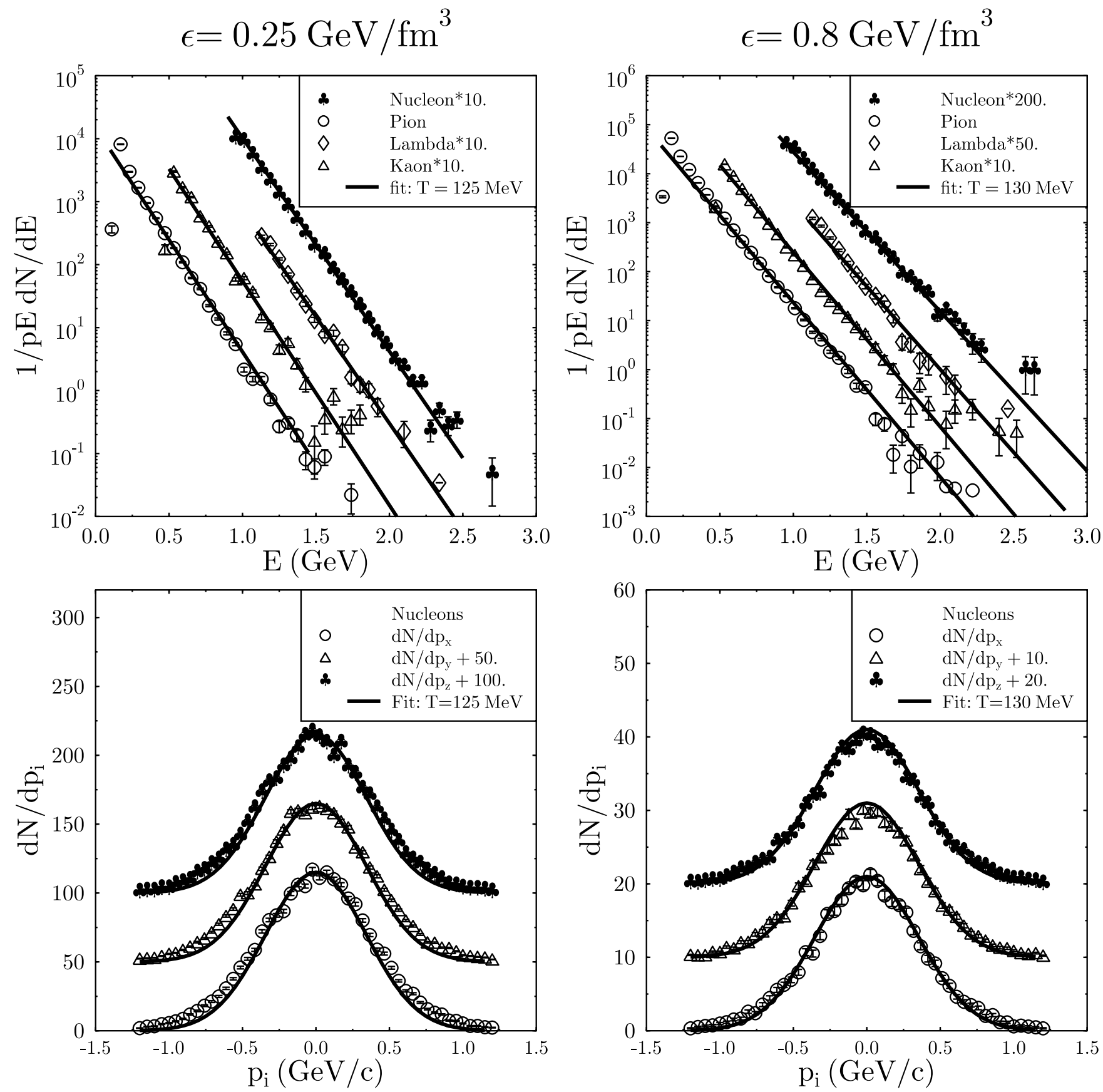

FIG. 2. Energy spectra (top panels) of nucleons, pions, lambdas and kaons, and momentum distributions (low panels) of nucleons at two different energy densities, $0.25 \mathrm{GeV} / \mathrm{fm}^{3}$ (left part) and $0.8 \mathrm{GeV} / \mathrm{fm}^{3}$ (right part). The calculations are done at $\rho_{B}=0.16 \mathrm{fm}^{-3}$ and $\rho_{S}=0 \mathrm{fm}^{-3}$. 


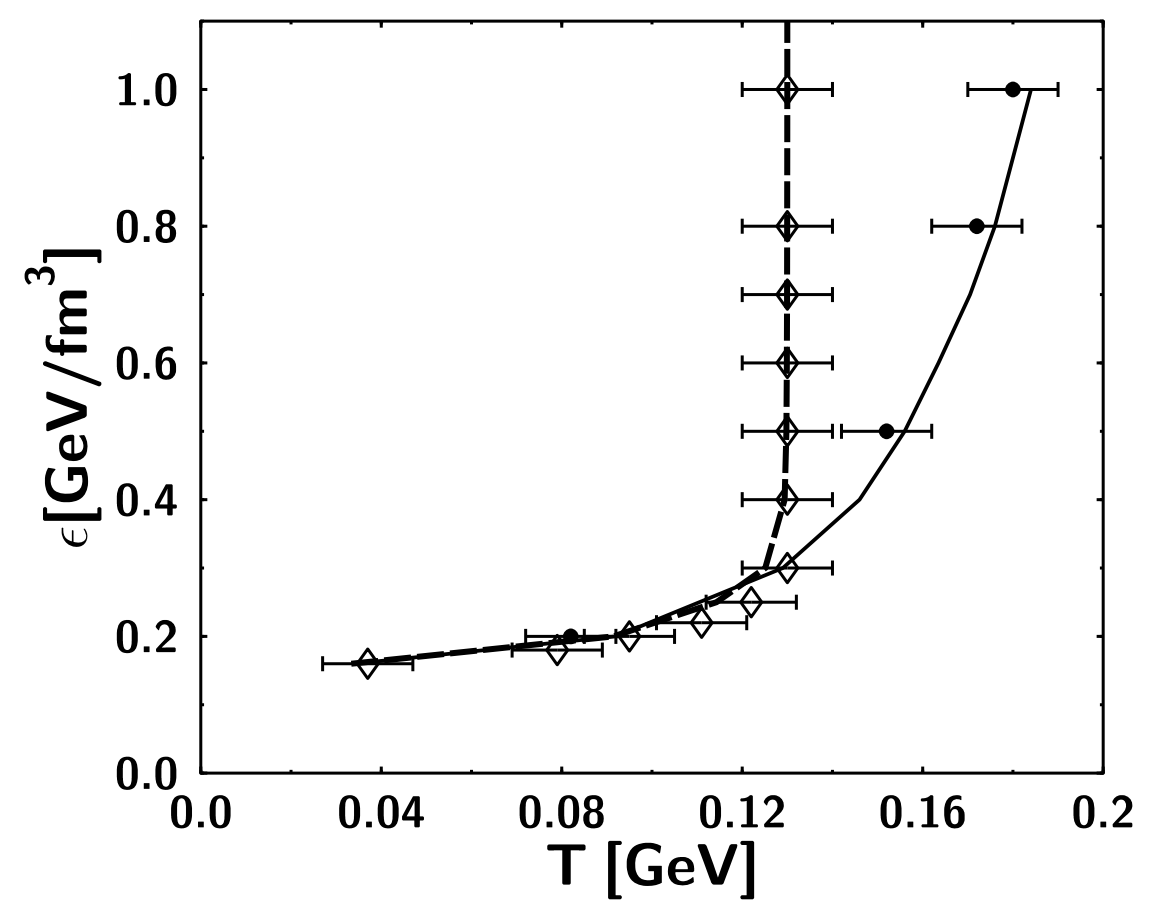

FIG. 3. Equation of State. The energy density $\epsilon$ is plotted versus the temperature $T$. The symbols show the results of UrQMD box calculations with (open diamonds) and without (solid circles) strings and many-body $(n>2)$ decays. The lines show the EoS of a quantum statistical model of ideal hadrons (solid line) plus continuous mass spectrum (dashed line) (see text). The calculations are done at $\rho_{B}=0.16 \mathrm{fm}^{-3}$ and $\rho_{S}=0 \mathrm{fm}^{-3}$. 

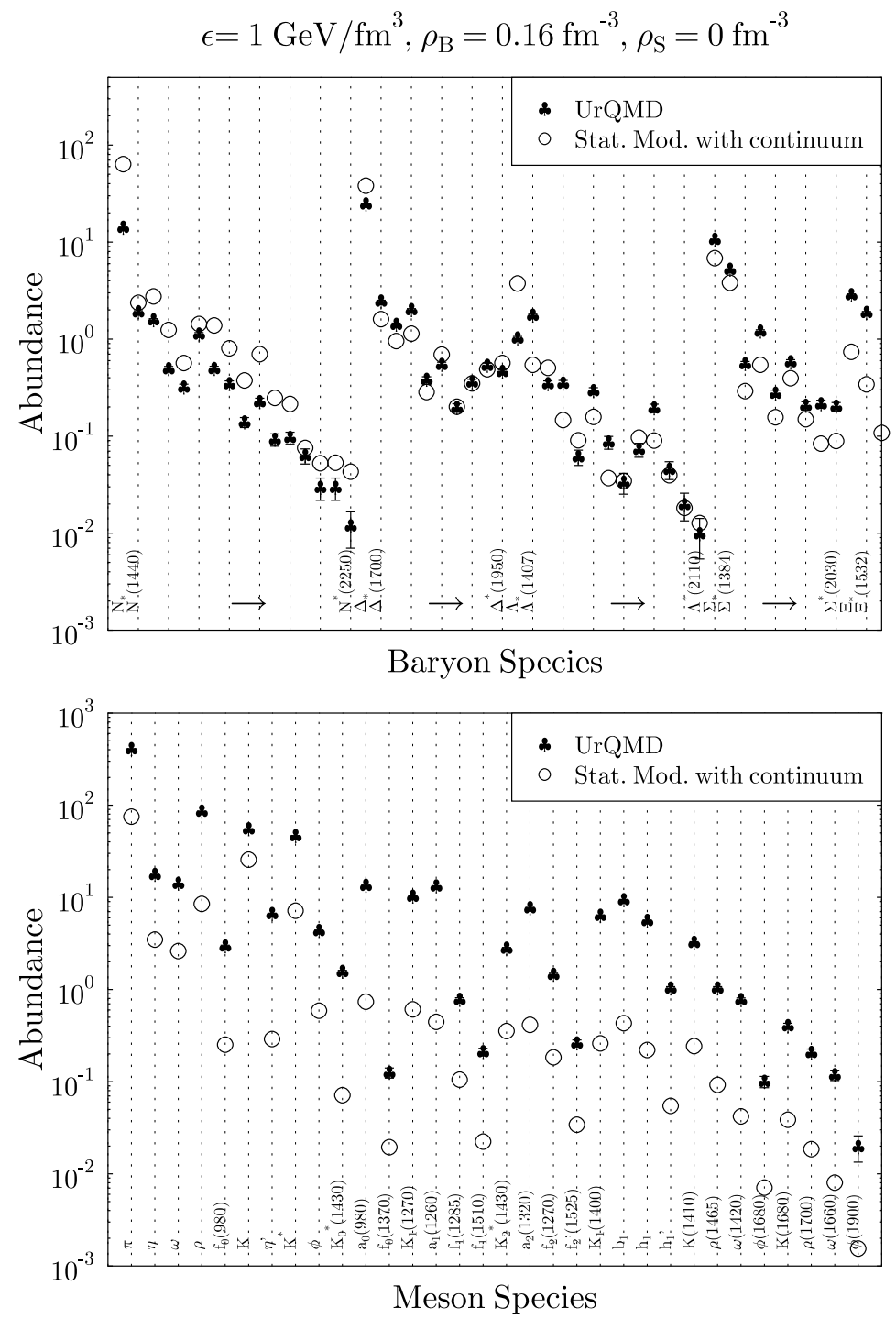

FIG. 4. Absolute particle abundances as obtained in UrQMD box calculations (club symbols) and in the statistical model (open circles) where high mass resonances from the continuum have been taken into account. 

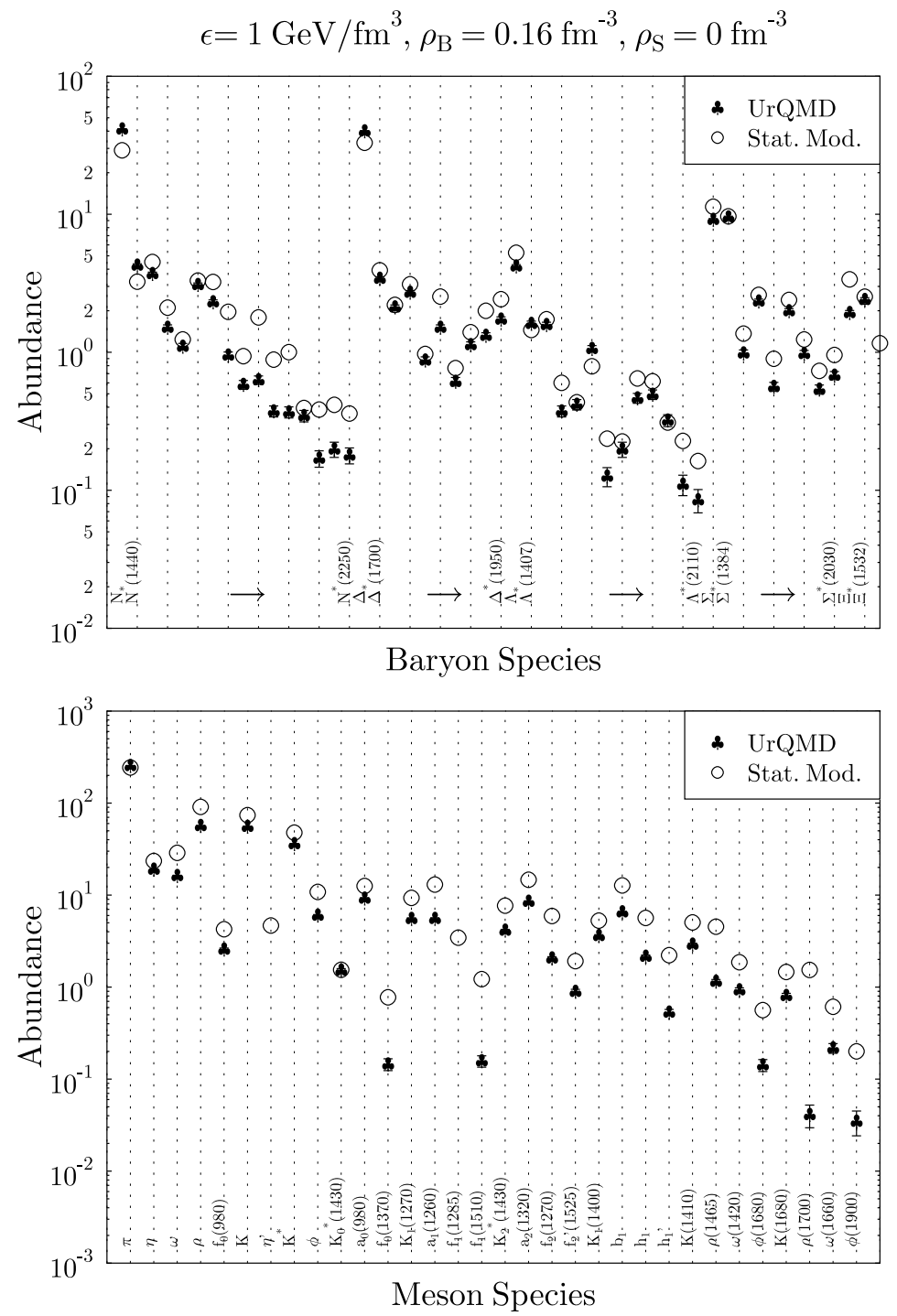

FIG. 5. Absolute particle abundances from UrQMD box calculations (club symbols) and from the statistical model (open circles). In UrQMD calculations, strings and many-body decays have been suppressed while no continuum has been taken into account in the statistical model. 


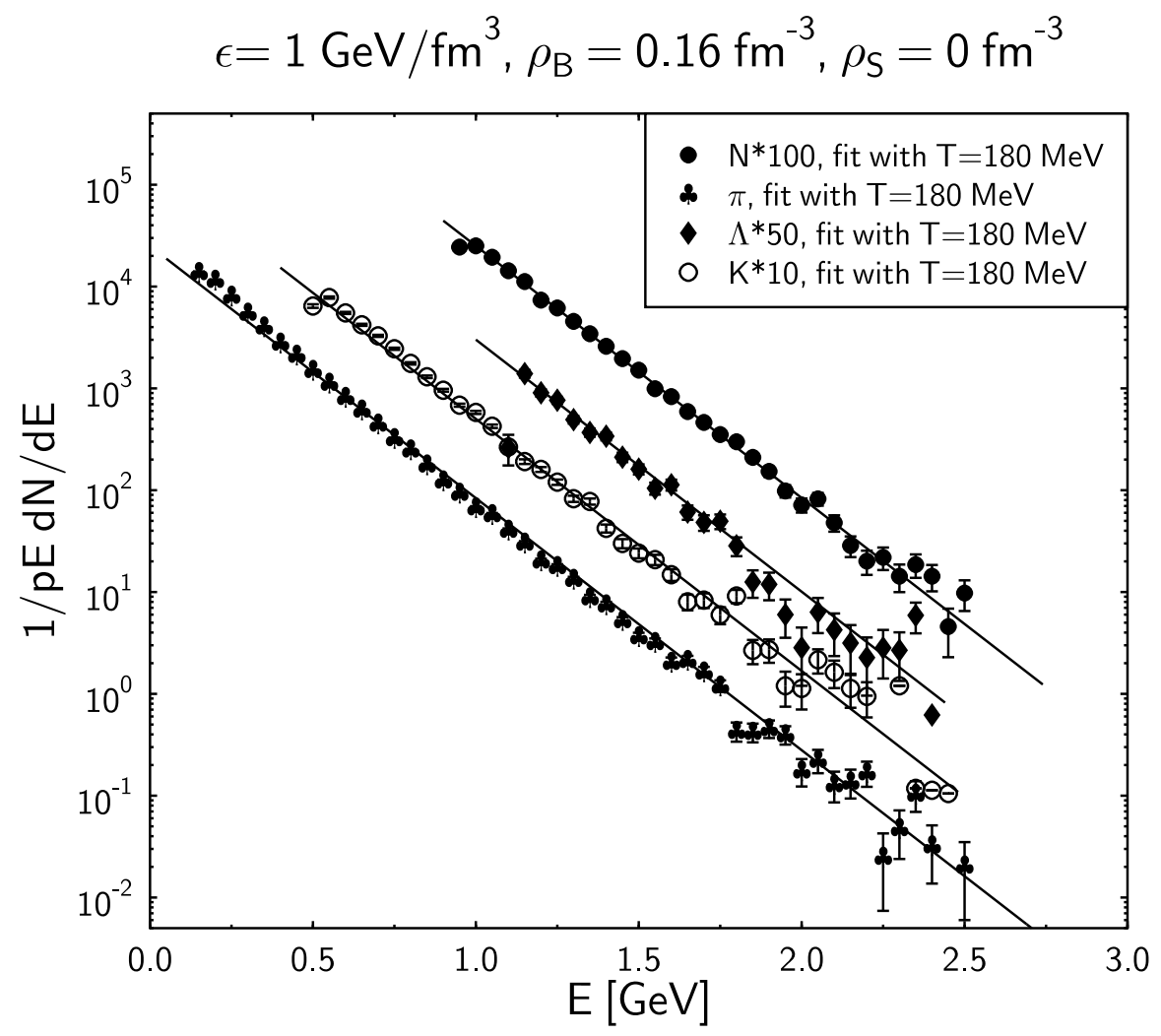

FIG. 6. Energy spectra for nucleons, pions, lambdas and kaons resulting from UrQMD box calculations without strings and many-body decays. All spectra are fitted by Boltzmann distributions with a single temperature $T=180 \mathrm{MeV}$. 